\title{
HEMIEUXOA POLYMORPHA FORBES 1933 PRIMER REGISTRO PARA CHILE: ¿RELICTO O DISPERSIÓN? (LEPIDOPTERA, NOCTUIDAE, NOCTUINAE)
}

\author{
HEMIEUXOA POLYMORPHA FORBES 1933 FIRST RECORD TO \\ CHILE: ¿RELICTUAL OR DISPERSAL? (LEPIDOPTERA, NOCTUIDAE, \\ NOCTUINAE)
}

\author{
Andrés O. Angulo ${ }^{1} \&$ Tania S. Olivares ${ }^{2}$ \\ ${ }^{1}$ Universidad de Concepción, Facultad de Ciencias Naturales y Oceanográficas, Departamento de Zoología, Casilla \\ 160-C. Concepción, Chile. Email: aangulo@udec.cl \\ ${ }^{2}$ Casilla 4040 correo 3. Concepción. Chile. E-mail: tolivare@udec.cl
}

\section{RESUMEN}

Se entrega el primer registro del lepidóptero nocturno Hemieuxoa polymorpha Forbes, 1933, para Chile. Adultos han sido recolectados en el sector Alto Patache, Iquique, Chile. Se mencionan las diferencias con la especie más próxima $H$. conchidia Butler.

Palabras claves: Lepidoptera, Noctuidae, Noctuinae, Alto Patache, Iquique, Chile, nuevo registro.

\section{ABSTRACT}

The first record of a noctuid moth Hemieuxoa polymorpha Forbes, 1933 for Chile is given. Adults have been collected in Alto Patache, Iquique, Chile. Differences between $H$. polymorpha Forbes and $H$. conchidia Butler are also given.

Keywords: Lepidoptera, Noctuidae, Alto Patache, Iquique, Chile, first record.

El género Hemieuxoa McDunnough [1929] 1928, es un género americano que se extiende desde el sur de Estados Unidos (Texas) hasta el sur del Perú (Lima); posee 6 especies (sensu Poole 1989): $H$. conchidia Butler 1882, H. interfascia Hampson 1918, H. interrupta Maasen 1890, H. nezia Schaus 1911, H. polymorpha Forbes 1933 y H. rudens Harvey 1875.

En 1999 durante el mes de noviembre en una recolección de mariposas nocturnas en el Norte de Chile, se encontraron adultos (1 macho y 1 hembra) de $H$. polymorpha en el Cerro Niebla de "Alto Patache" (20 49'S 70 09' W) a $65 \mathrm{~km}$ al sur de Iquique, lo que constituye el primer registro para
Chile y a la vez aumenta el rango de distribución de esta especie en Sudamérica; anteriormente su registro más meridional había sido la parte desértica del sur del Perú en las localidades de Lima, Chosica y Matucana (Forbes 1933).

Como la especie se encuentra en el sur del Perú y ahora ha sido encontrada en Iquique (Cerro Niebla Alto Patache), existe un discontinuidad de la distribución, lo que representa un relicto de la distribución de la especie o bien una colonización reciente de este nuevo hábitat más húmedo y de más alta latitud; ello podrá ser dilucidado al continuar recolectando en los sitios intermedios entre Iquique y sur del Perú. 
Material examinado: $10^{*}$ y 19 , Chile, I Región, provincia de Tarapacá, Iquique, Alto Patache 860 m., 7-11-1999, Angulo Coll.(gen prep.) (UCCC). La especie más próxima a $H$. polymorpha es $H$. conchidia Butler y sus diferencias son:
Finalmente se concluye que en Chile se encuentran presentes dos especies del género Hemieuxoa: H. conchidia Butler, que se distribuye desde Tarapacá hasta Concepción y H. polymorpha Forbes

\begin{tabular}{cll}
\hline \multicolumn{1}{c}{ Caracteres } & H. conchidia & H. polymorpha \\
\hline Adulto: mancha reniforme & Con escamas rojizas & Casi sin presencia de \\
& amarillentas & escamas rojizas amarillentas \\
Genitalia macho: ampulla & Redondeada, en forma de & Alargada, digitiforme \\
& tubérculo & \\
aedeagus & Con dos espinas & Con una espina \\
\hline
\end{tabular}

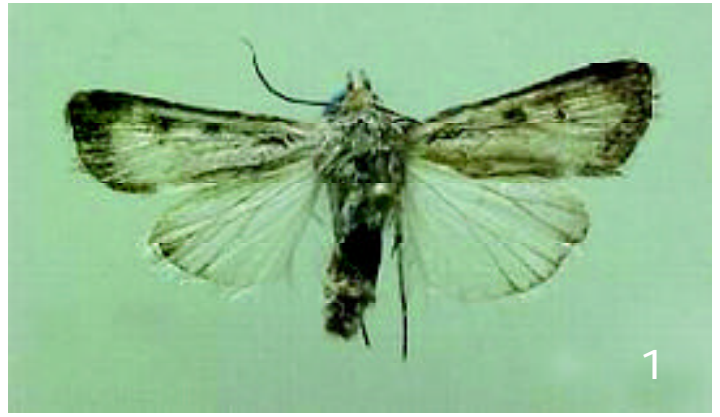

Figura 1. Adulto de H. conchidia Butler.

Figure 1. Adult of $H$. conchidia Butler

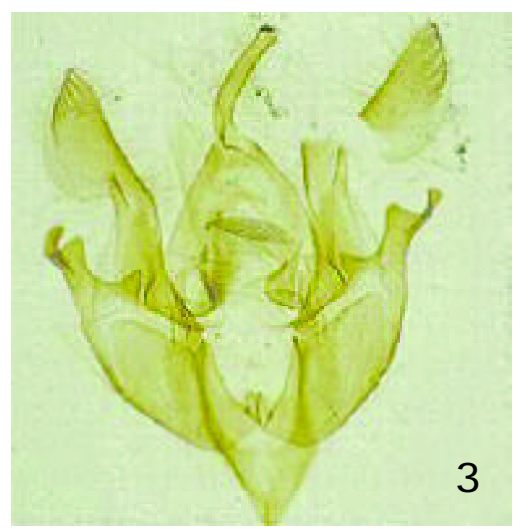

FIgURA 3. Genitalia del macho de $H$. conchidia Butler.

FIGURA 3. Male genitalia of $H$. conchidia Butler

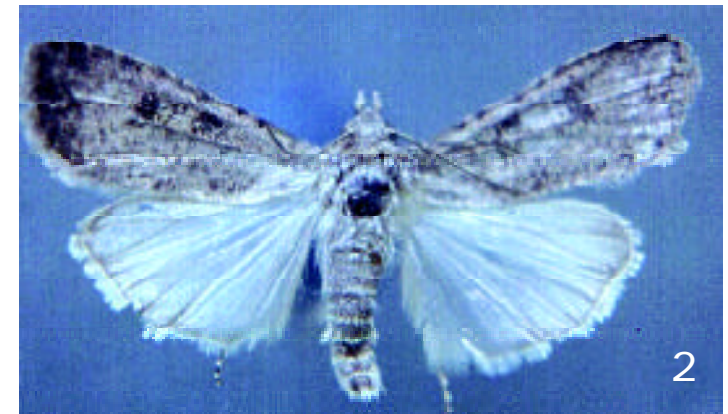

FIGURA 2. Adulto de H. polymorpha Forbes.

Figure 2. Adult of $H$. polymorpha Forbes

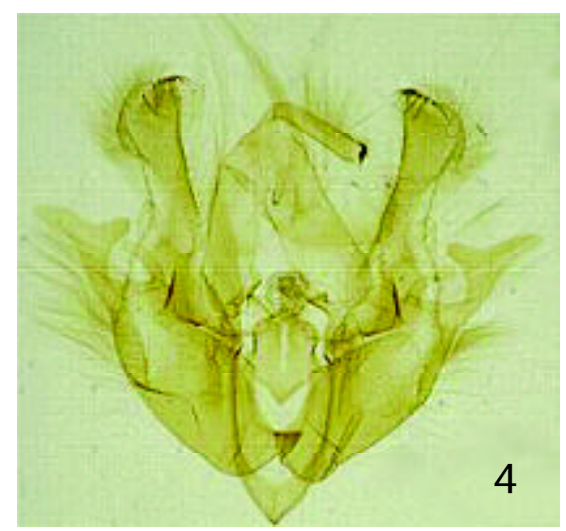

Figura 4. Genitalia del macho de H. polymorpha Forbes.

FIGURE 4. Male genitalia of $H$. polymorpha Forbes 


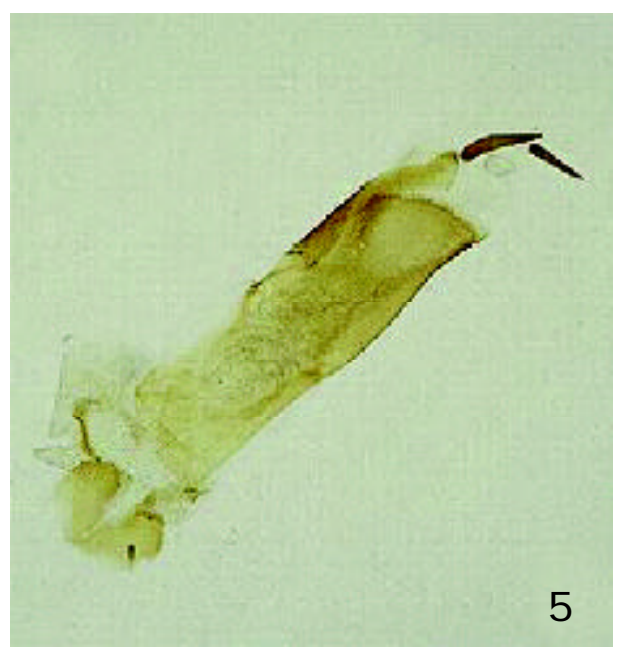

Figura 5. Aedeagus de $H$. conchidia Butler.

Figure 5. Aedeagus of $H$. conchidia Butler.

\section{AGRADECIMIENTOS}

Deseamos agradecer al Proyecto de Investigación 203.113.060-1.0. A Horacio Larraín y Marta Peña por facilitar el acceso al lugar de muestreo.

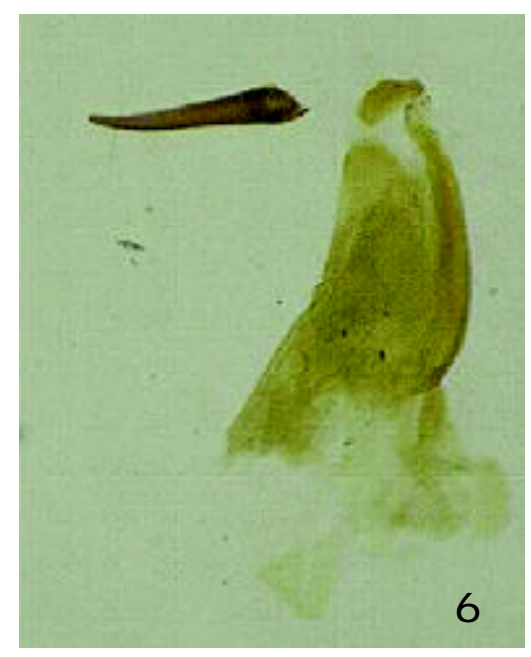

Figura 6. Aedeagus de H. polymorpha Forbes.

FIGURE 6. Aedeagus of $H$. polymorpha Forbes.

\section{BIBLIOGRAFIA}

Forbes, T.M. 1933. A grouping of the Agrotinae genera. Entomologica Americana. 14(1):1-30 pp.+ 6 plates.

Poole, R.W. 1989. Lepidopterorum Catalogus. (New Series). Fasc. 118 Noctuidae. Part 1. E.J. Brill /Flora and Fauna Publications. 